ARTICLE

Received 12 Sep 2016 | Accepted 4 Jan 2017 | Published 19 Apr 2017 DOl: 10.1038/ncomms14474

\title{
Efficient electrical control of thin-film black phosphorus bandgap
}

Bingchen Deng ${ }^{1, \star}$, Vy Tran ${ }^{2, \star}$, Yujun Xie ${ }^{3}$, Hao Jiang ${ }^{4}$, Cheng Li ${ }^{1}$ Qiushi Guo ${ }^{1}$ Xiaomu Wang ${ }^{1}$, He Tian ${ }^{5}$, Steven J. Koester ${ }^{6}$, Han Wang ${ }^{5}$, Judy J. Cha ${ }^{3}$, Qiangfei Xia ${ }^{4}$, Li Yang$^{2}$ \& Fengnian Xia ${ }^{1}$

Recently rediscovered black phosphorus is a layered semiconductor with promising electronic and photonic properties. Dynamic control of its bandgap can allow for the exploration of new physical phenomena. However, theoretical investigations and photoemission spectroscopy experiments indicate that in its few-layer form, an exceedingly large electric field in the order of several volts per nanometre is required to effectively tune its bandgap, making the direct electrical control unfeasible. Here we reveal the unique thickness-dependent bandgap tuning properties in intrinsic black phosphorus, arising from the strong interlayer electronic-state coupling. Furthermore, leveraging a $10 \mathrm{~nm}$-thick black phosphorus, we continuously tune its bandgap from $\sim 300$ to below $50 \mathrm{meV}$, using a moderate displacement field up to $1.1 \mathrm{Vnm}^{-1}$. Such dynamic tuning of bandgap may not only extend the operational wavelength range of tunable black phosphorus photonic devices, but also pave the way for the investigation of electrically tunable topological insulators and semimetals.

\footnotetext{
${ }^{1}$ Department of Electrical Engineering, Yale University, 15 Prospect St Becton 519, New Haven, Connecticut 06511, USA. ${ }^{2}$ Department of Physics, Washington University, St Louis, Missouri 63130, USA. ${ }^{3}$ Department of Mechanical Engineering and Materials Science, Yale University, New Haven, Connecticut 06511, USA. ${ }^{4}$ Department of Electrical and Computer Engineering, University of Massachusetts, Amherst, Massachusetts 01003, USA. ${ }^{5}$ Ming Hsieh Department of Electrical Engineering, University of Southern California, Los Angeles, California 90089, USA. ${ }^{6}$ Department of Electrical and Computer Engineering, University of Minnesota, Minneapolis, Minnesota 55455, USA. * These authors contributed equally to this work. Correspondence and requests for materials should be addressed to L.Y. (email: lyang@physics.wustl.edu) or to F.X. (email: fengnian.xia@yale.edu).
} 
B andgap is a fundamental material parameter that governs the transport and light-matter interaction properties. Black phosphorus (BP) lately emerged as a promising layered material ${ }^{1-10}$, which bridges the gap between the gapless graphene ${ }^{11,12}$ and semiconducting transition metal dichalcogenides (TMDCs) ${ }^{13}$ such as molybdenum disulfide $\left(\mathrm{MoS}_{2}\right)$ with a relatively large bandgap of around $2 \mathrm{eV}$. Owing to the strong electronic state coupling among layers in BP, its direct bandgap varies significantly from around $0.3 \mathrm{eV}$ in bulk to $2 \mathrm{eV}$ in monolayer form ${ }^{9}$. On the other hand, an approach that can dynamically tune the bandgap of BP to well below $0.3 \mathrm{eV}$ is of great scientific and technological importance. In fact, the feasibility of bandgap tuning in few-layer BP using an electric field has been explored theoretically ${ }^{14-17}$. Recently, photoemission spectroscopy experiments in doped few-layer BP through the adsorption of potassium atoms show that indeed its bandgap can be tuned and even completely closed ${ }^{18}$. However, the estimated electric field induced by potassium adsorption in few-layer BP to widely tune its bandgap is on the order of several volts per nanometre. Such a large electric field prevents the realization of bandgap tunable electronic and photonic devices. Moreover, heavily doped material is usually not ideal for device applications. As a result, it is highly attractive to realize a widely tunable bandgap in intrinsic BP with a moderate field accessible with regular dielectrics.

In this work, we demonstrate bandgap tuning in BP using an electric field directly and discover unique thickness-dependent bandgap tuning properties, guided by first-principles calculations. In a $4 \mathrm{~nm}$-thick intrinsic BP film, the bandgap tuning is limited to around $75 \mathrm{meV}$ and shows strong nonlinear dependence on the biasing field. This peculiar field dependence is due to the strong interlayer electronic-state coupling and is captured by density functional theory (DFT) calculations very well. On the contrary, in a $10 \mathrm{~nm}$-thick intrinsic BP in which the film is thick enough such that the field-induced potential difference can overcome the interlayer coupling, we demonstrate the efficient, continuous tuning of its bandgap from $\sim 300$ to below $50 \mathrm{meV}$ with an external displacement field up to $1.1 \mathrm{~V} \mathrm{~nm}^{-1}$. We further show that in this case, the additional dielectric screening effect beyond a simple DFT prediction due to the significant bandgap shrinkage has to be taken into account to describe the experimental results.

\section{Results}

Device fabrication and basic characterizations. We use dual-gate BP transistors as shown in Fig. 1a for the bandgap measurement under a bias field. Here, the thin-film BP channel is sandwiched between the back ( $90 \mathrm{~nm}$-thick silicon oxide) and top ( $24 \mathrm{~nm}$-thick aluminum oxide) gate dielectrics. The source and drain leads are made from chromium/gold $(3 / 30 \mathrm{~nm})$. The top gate is made from titanium/platinum $(1 / 10 \mathrm{~nm})$ and the silicon substrate is used as the back gate. The bandgap is determined through the $\mathrm{BP}$ conductance at the charge neutrality point under a vertical biasing field. To eliminate the impact of metal/BP contact on the conductance measurement, a four-probe scheme is adopted, as shown in Fig. 1a. In our measurements, the source is grounded and the drain bias $V_{\mathrm{D}}$ is kept at $100 \mathrm{mV}$. From the channel current and the voltage difference between probes 1 and 2, we determine the conductance of the BP film. The detailed device fabrication and measurement procedures are discussed in the Methods section. In Fig. 1b, the measured conductance $G_{12}$ of a $4 \mathrm{~nm}$-thick BP flake between probes 1 and 2 (see inset) at room temperature is plotted as a function of the back gate bias, $V_{\mathrm{BG}}$, whereas the top gate bias, $V_{\mathrm{TG}}$, is set to be 0 volt. The conductance is plotted in both linear (left axis) and
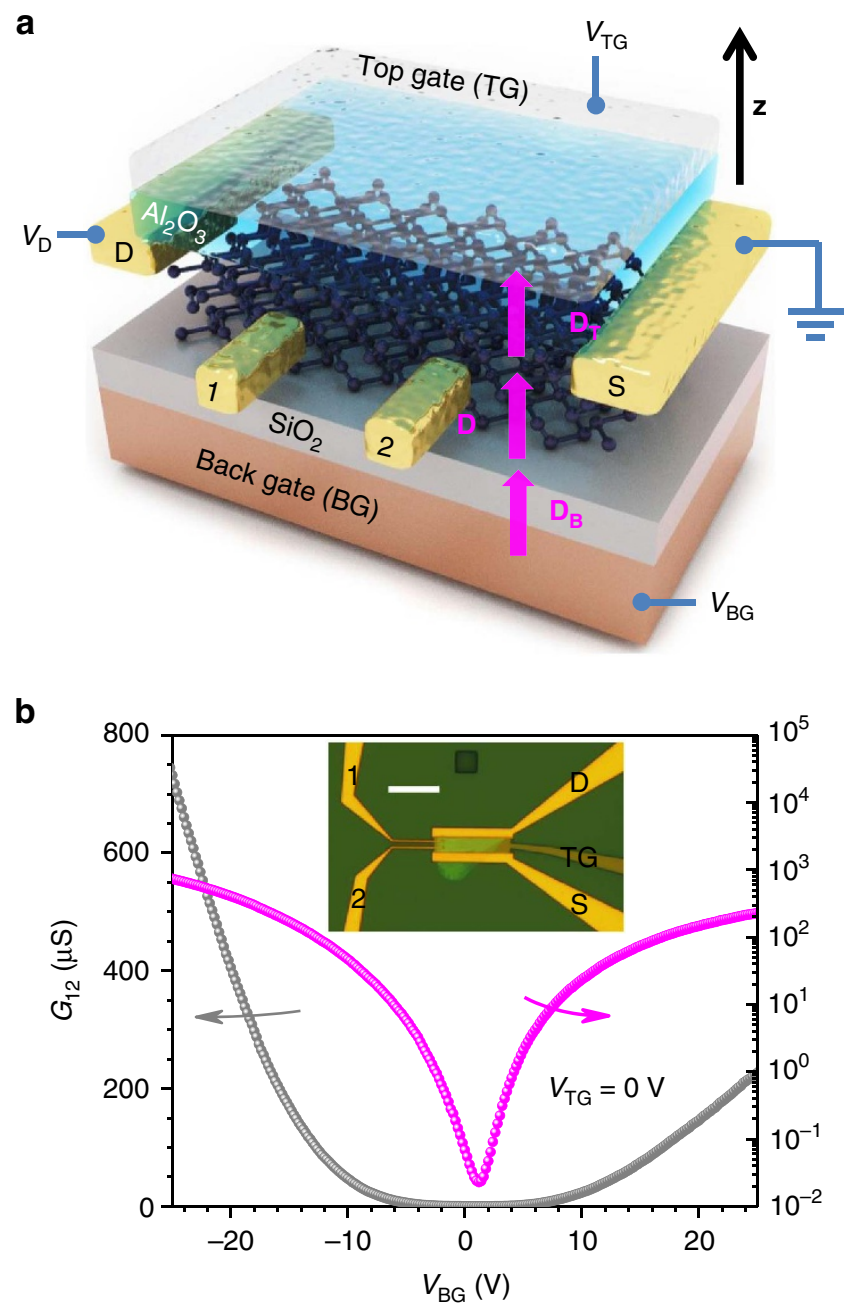

C

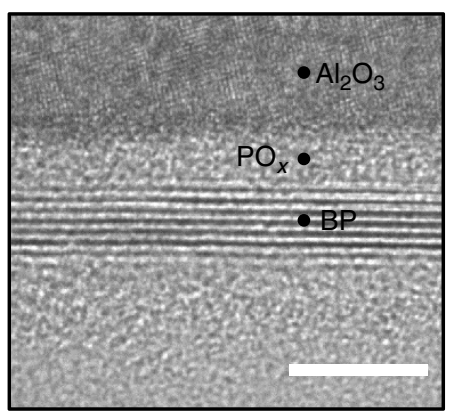

d

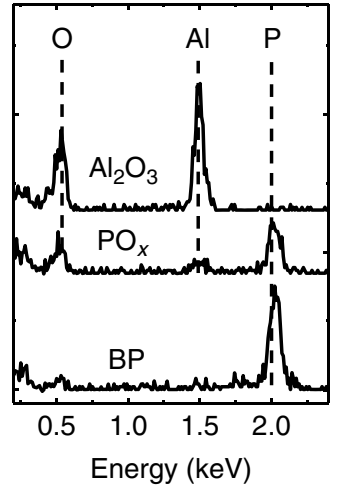

Figure 1 | Experimental scheme for BP bandgap tuning. (a) The schematic view of the dual-gate BP thin-film transistor for the realization of bandgap tuning. (b) The $4 \mathrm{~nm}$-thick BP film conductance in linear (left axis) and logarithmic (right axis) scales as a function of the back gate bias $\left(V_{\mathrm{BG}}\right)$ at zero top gate bias $\left(V_{\mathrm{TG}}=0 \mathrm{~V}\right)$. The conductance $G_{12}$ is measured using a four-probe scheme as shown in $\mathbf{a}$, to eliminate the effects of the contact resistance. Inset: an optical image of a typical dual-gate BP transistor. Scale bar, $10 \mu \mathrm{m}$. (c) High-resolution cross-section transmission electron micrograph showing the $\mathrm{BP}$ layer, which is about $4 \mathrm{~nm}$ thick. The $\mathrm{PO}_{x}$ layer between the $\mathrm{BP}$ layer and $\mathrm{Al}_{2} \mathrm{O}_{3}$ is $2-3 \mathrm{~nm}$ thick. Scale bar, $8 \mathrm{~nm}$. (d) Energy dispersive $\mathrm{X}$-ray spectra from three selected spots in $\mathbf{c}$. 
logarithmic (right axis) scales. As the bandgap of BP is moderate, the conductance in the insulating state at room temperature can still be measured using a four-probe scheme, as shown by the logarithmic plot (right axis, Fig. 1b). Here the BP thickness is determined using the atomic force microscopy (AFM) together with the cross-section high-resolution transmission electron microscopy, as shown in Supplementary Fig. 1 and Fig. 1c, respectively. Energy dispersive X-ray spectra from three selected spots in Fig. 1c are plotted in Fig. 1d. It is rather clear that the phosphorus oxide $\left(\mathrm{PO}_{x}\right)$ at the top $\mathrm{BP}$ surface is $2-3 \mathrm{~nm}$ thick due to the exposure to external environment during processing. However, at the bottom $\mathrm{BP} / \mathrm{SiO}_{2}$ interface as shown in Fig. 1c, there is no obvious sign of BP oxidization, because the sample exfoliation was performed in the glove box. As a result, the intrinsic BP thickness in our devices is always $2-3 \mathrm{~nm}$ less than the value measured using AFM, as discussed in Supplementary Note 1. In fact, our previous generations of $\mathrm{BP}$ devices have thicker $\mathrm{PO}_{x}$ on both top and bottom surfaces due to the exfoliation in ambient environment and lack of top gate dielectric protection.

Bandgap tuning in a $4 \mathbf{n m}$-thick BP film. We further performed conductance measurements in such dual-gated BP transistors by sweeping the top gate bias at different static back gate biases. The measurement results for a BP transistor with a channel thickness of around $4 \mathrm{~nm}$ are summarized in Fig. 2a. As shown in the inset of Fig. $2 \mathrm{~b}$, the top gate bias $\left(V_{\mathrm{TG}}\right)$ at which the conductance is minimized $\left(V_{\mathrm{TM}}\right)$ scales almost linearly with the back gate bias $\left(V_{\mathrm{BG}}\right)$. This observation indicates that the top gate bias with opposite sign can effectively compensate the doping introduced by the back gate at the $V_{\mathrm{TG}}=V_{\mathrm{TM}}$, leading to an insulating state at the charge neutrality point. As the source is grounded and a very small drain bias of $100 \mathrm{mV}$ is applied, the displacement fields in the back $\left(\mathbf{D}_{\mathrm{B}}\right)$ and top $\left(\mathbf{D}_{\mathrm{T}}\right)$ gate dielectric are $\mathbf{D}_{\mathrm{B}}=\varepsilon_{\mathrm{B}} \frac{V_{\mathrm{BG}}}{d_{\mathrm{B}}} \mathbf{z}$ and $\mathbf{D}_{\mathrm{T}}=-\varepsilon_{\mathrm{T}} \frac{V_{\mathrm{TG}}}{d_{\mathrm{T}}} \mathbf{z}$, respectively, where $\varepsilon_{\mathrm{B}}(\sim 3.9)$ is the relative permittivity of $\mathrm{SiO}_{2}, d_{\mathrm{B}}(90 \mathrm{~nm})$ is the thickness of $\mathrm{SiO}_{2}, \varepsilon_{\mathrm{T}}$ is the overall relative permittivity of top gate dielectric, $d_{\mathrm{T}}(26 \mathrm{~nm})$ is the total thickness of top gate dielectric and $\mathbf{z}$ is the unit vector along the vertical direction as shown in Fig. 1a. Here, displacement is defined as positive when it points from back towards the top gates. The $d_{\mathrm{T}}$ includes the $2 \mathrm{~nm}$-thick $\mathrm{PO}_{x}$ layer and $24 \mathrm{~nm}$-thick $\mathrm{Al}_{2} \mathrm{O}_{3}$. From the slope of the curve in the inset of Fig. $2 \mathrm{~b}, \varepsilon_{\mathrm{T}}$ is determined to be $\sim 5.6$. At the charge-neutrality condition $\left(V_{\mathrm{TG}}=V_{\mathrm{TM}}\right), \mathbf{D}_{\mathrm{B}}=\mathbf{D}_{\mathrm{T}}=\mathbf{D}$, where $\mathbf{D}$ is the displacement field in BP channel, as shown in Fig. 1a. We assume that at the charge-neutrality condition, the free carrier concentration in BP is minimized such that $\mathbf{D}$ is approximately uniform across the entire channel. For $100 \mathrm{mV}$ source-drain bias, the maximum possible displacement variation between the BP and the top gate along the channel is estimated to be $<0.02 \mathrm{~V} \mathrm{~nm}^{-1}$, much smaller than the field we are interested in $\left(\sim 0.2-1.1 \mathrm{~V} \mathrm{~nm}^{-1}\right)$. As a result, the effect of finite source-drain bias on the vertical displacement field is negligible in this work.

In Fig. 2b, the minimal conductance at the charge-neutrality point is plotted as a function of the displacement field $\mathbf{D}$ in BP. It increases by $\sim 5$ times at maximum bias field, clearly indicating a reduction of the energy gap. We quantify this bandgap reduction in the following. The minimum conductivity $\sigma_{\mathrm{m}}$ at the charge neutrality can be calculated using

$$
\sigma_{\mathrm{m}}=q n_{\mathrm{i}}\left(\mu_{\mathrm{e}}+\mu_{\mathrm{h}}\right)
$$

where $q$ is the elementary electron charge, $n_{\mathrm{i}}$ is the intrinsic, thermally excited carrier density for both electrons and holes at charge neutrality, and $\mu_{\mathrm{e}}$ and $\mu_{\mathrm{h}}$ are the mobility for electrons and holes, respectively. If the bandgap reduction is much smaller than the bandgap itself, we can assume that the band structure
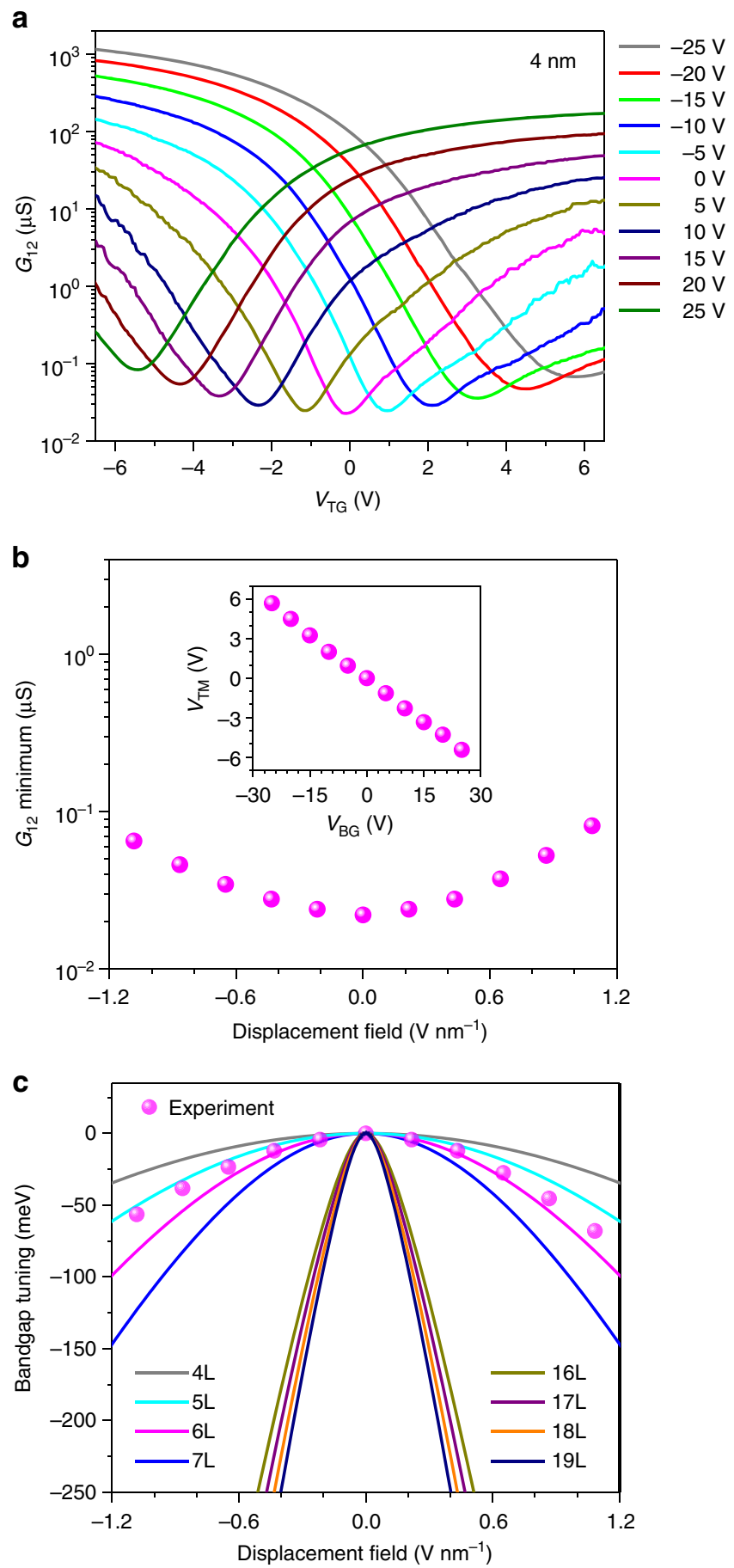

Figure 2 | Bandgap tuning in a $\mathbf{4} \mathbf{n m}$-thick BP film. (a) The $4 \mathrm{~nm}$-thick BP film conductance as a function of top gate bias $\left(V_{\mathrm{TG}}\right)$ at different static back gate biases $\left(V_{\mathrm{BG}}\right)$ from -25 to $25 \mathrm{~V}$. (b) The minimum conductance at the charge-neutrality point as a function of external displacement field. Inset: the top gate bias at which the minimum conductance occurs $\left(V_{T M}\right)$ as a function of the back gate bias $\left(V_{B G}\right)$. The linear relation indicates the effective doping compensation by the back and top biases, leading to an insulating state at the charge-neutrality. (c) Solid lines: The calculated bandgap tuning properties for BP films consisting of 4, 5, 6, 7, 16, 17, 18 and 19 layers using a tight-binding model built on the DFT. The BP films with different thicknesses exhibit distinctively different tuning properties. Purple dots: the measured bandgap tuning results for the $4 \mathrm{~nm}$-thick BP film ( 7 layers $)$. 
does not change and hence the carrier mobility remains unchanged. In this case, from the minimum conductivity variation, we can estimate the bandgap reduction, as $n_{\mathrm{i}}$ is proportional to $\mathrm{e}^{-\frac{E_{\mathrm{g}}}{2 k_{\mathrm{B}} T}}$, where $E_{\mathrm{g}}$ is the bandgap, $k_{\mathrm{B}}$ is the Boltzmann constant and $T$ is temperature $(295 \mathrm{~K})^{19}$. The purple dots in Fig. 2c indicate the estimated bandgap shrinkage as a function of the externally applied displacement field, which does not exceed $75 \mathrm{meV}$ and shows a strong nonlinear dependence. Indeed, the reduction is much smaller than the quasi-particle bandgap of $\sim 4 \mathrm{~nm}$-thick BP ( $\sim 7$ layers), which should be around $450 \mathrm{meV}^{9}$.

We have performed theoretical calculations using DFT, to explore the bandgap tuning effect in BP thin film. Based on the DFT results, we have further developed a tight-binding model (see details in the Supplementary Note 2, Supplementary Figs 2 and 3, and Supplementary Table 1), in which the interlayer coupling $(\delta)$ is introduced in the off-diagonal terms of the Hamiltonian and the screen field-induced potential difference $(\Delta)$ between layers is placed in the diagonal terms. This model can reliably reproduce DFT results for few-layer BP and enable us to efficiently calculate much thicker BP that is beyond the DFT capability. As shown in Fig. 2c, our experimental results measured on a $4 \mathrm{~nm}$-thick BP sample (approximately seven layers nominally) fall in between the theoretical five- and six-layer results. Most importantly, the theoretical results capture the nonlinear character of the bandgap evolution as the gating field increases. In short, the bandgap tuning effect arises from the overall potential difference induced by the external gating field. However, for ultra-thin (few-layer) BP films, the potential drop is less than the interlayer coupling (off-diagonal term $\delta$ ) under low bias. Thus, the bandgap tuning exhibits a very strong nonlinear dependence on the external bias field and the tunable range is rather limited with a moderate displacement field up to $\sim 1 \mathrm{~V} \mathrm{~nm}^{-1}$. On the contrary, our model predicts that, when the thickness is large enough, the total potential difference across the entire BP thin film dominates, leading to more effective and almost linear dependence of the tuning with the applied bias field as shown in Fig. 2c for thicker BP (16-19 layers). Although in general for thicker material the bandgap tuning effect should be larger, because the Stark coefficient scales with thickness ${ }^{17,18}$, here we reveal the distinctively different tuning properties of BP films whose thicknesses fall into two different categories as shown in Fig. 2c.

Bandgap tuning in a $10 \mathrm{~nm}$-thick BP film. Therefore, to extend the bandgap tuning range, we further fabricated the dual-gate transistors with BP channel thickness of $\sim 10 \mathrm{~nm}$. The conductance measurement results similar to those in $4 \mathrm{~nm}$-thick transistors are plotted in Fig. 3a. The minimum conductance at the charge-neutrality point as a function of the external displacement field is plotted in Fig. 3b. In this transistor, the minimum conductance at the charge neutrality increases by around 40 times as the external bias is maximized, indicating a bandgap reduction significantly $>75 \mathrm{meV}$. In this case, direct extraction of the bandgap variation from the minimum conductance at different biasing field may not be optimal, as the bandstructure itself can vary as the biasing changes the bandgap significantly. In this transistor, we measured the temperature dependence of the minimum conductance to determine the energy gap. Using this method, we not only eliminate the effect of bandstructure change on bandgap determination, but also measure the bandgap directly. The measurement results for the back gate biases of $-15,0$ and $15 \mathrm{~V}$ are shown in Fig. $4 \mathrm{a}$. In Fig. $4 \mathrm{~b}$, similar curves for the back gate biases of -25 and $25 \mathrm{~V}$ are shown. The detailed bandgap extraction procedures and
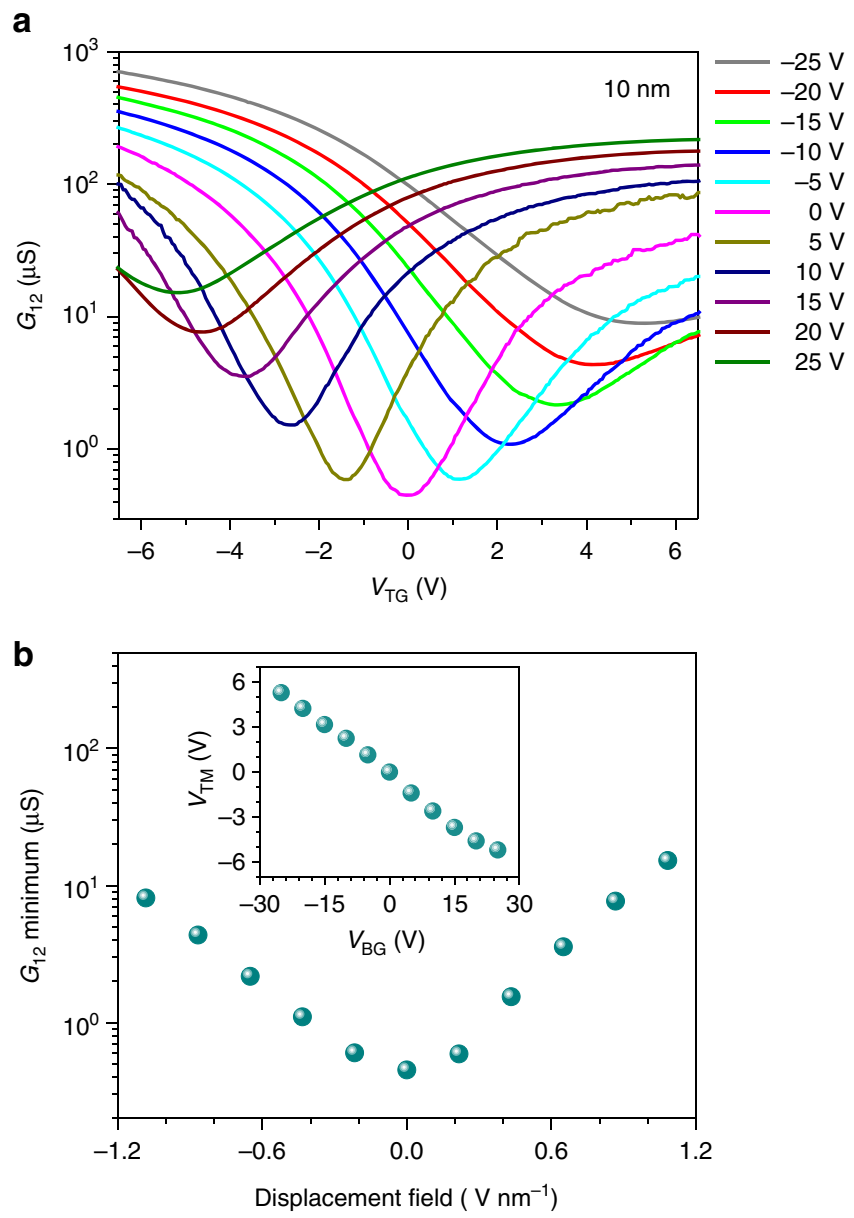

Figure 3 | Bandgap tuning in a 10 nm-thick BP film. (a) The 10 nm-thick BP film conductance as a function of top gate bias $\left(V_{\mathrm{TG}}\right)$ at different static back gate biases $\left(V_{\mathrm{BG}}\right)$ from -25 to $25 \mathrm{~V}$. (b) The minimum conductance at the charge-neutrality point as a function of external displacement field. Inset: the top gate bias at which the minimum conductance occurs $\left(V_{\mathrm{TM}}\right)$ as a function of the back gate bias $\left(V_{B G}\right)$.

the typical fitting curves are presented in the Supplementary Note 3 and Supplementary Figs 4 and 5. Below $120 \mathrm{~K}$, the BP film at zero bias becomes highly insulating and it is not feasible to measure its conductance. The minimum conductance at zero bias varies by around four orders of magnitude from 120 to $295 \mathrm{~K}$ as shown in Fig. 4a, from which a bandgap of $290 \mathrm{meV}$ is extracted. This value is close to the established bulk BP bandgap of $\sim 300 \mathrm{meV}$, indicating the accuracy of the approach used here. The slight discrepancy can be due to the intrinsic fitting errors of this approach, as the bandgap of BP itself does change by tens of $\mathrm{meV}$ as the temperature varies from 300 to $120 \mathrm{~K}$. When the applied displacement field increases from 0 to $1.1 \mathrm{~V} \mathrm{~nm}^{-1}$ ( $V_{\mathrm{BG}}$ positive and $V_{\mathrm{TG}}$ negative), the bandgap is measured to decrease from 290 to $30 \mathrm{meV}$ continuously, as shown in Fig. 4c. Reversing the direction of the displacement field leads to similar reduction of $\mathrm{BP}$ bandgap. When the temperature changes, the top gate bias at which the minimum conductance occurs, $V_{\mathrm{TM}}$, varies slightly $(<0.5 \mathrm{~V})$. We hence introduced an error bar to account for this uncertainty in external biasing displacement field in Fig. 4c.

In Fig. 4c, we also plot the calculated bandgap tuning results using tight-binding model built on DFT results for BP thickness from 9 to $11 \mathrm{~nm}$ ( $\sim 17$ to 21 layers), as shown by the dashed lines. It is clear that DFT predicts a faster bandgap tuning rate as a function of the biasing field. This discrepancy is not surprising, 
a

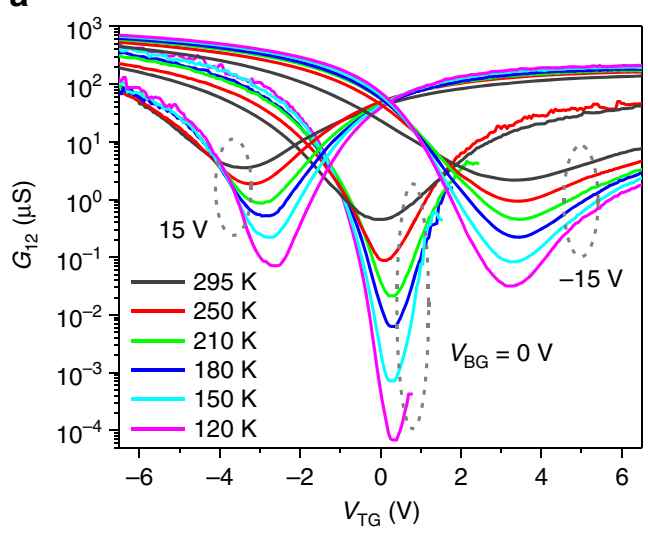

b

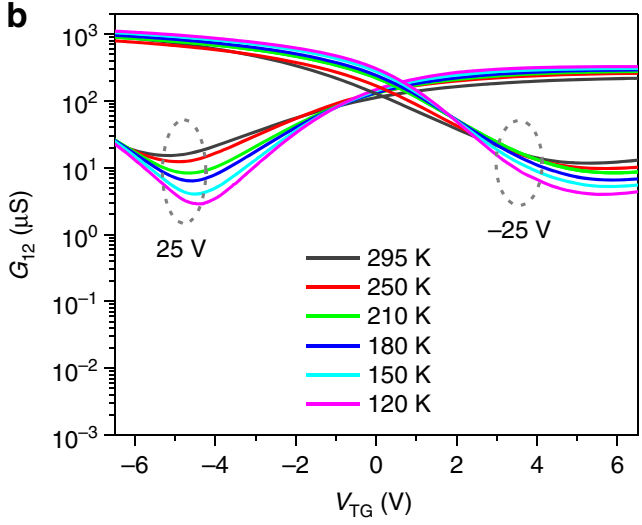

C

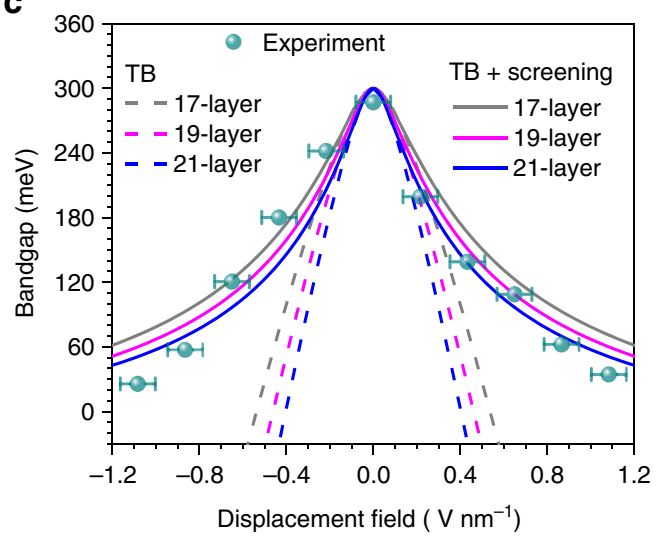

Figure 4 | Bandgap determination in $10 \mathrm{~nm}$-thick BP using temperaturedependent measurements. (a) The conductance of the $10 \mathrm{~nm}$-thick BP film as a function of top gate bias $\left(V_{\mathrm{TG}}\right)$ at different temperatures from 120 to $295 \mathrm{~K}$. The curves at three different back gate biases are plotted $\left(V_{B G}=0,-15\right.$ and $\left.15 \mathrm{~V}\right)$. The bandgap is measured through the temperature-dependent minimum conductivity at the charge-neutrality point. (b) Conductance measurement curves similar to those in a at two different back gate biases $\left(V_{B G}=-25\right.$ and $25 \mathrm{~V}$ ). (c) Dash (solid) lines: the calculated bandgap tuning properties for BP films consisting of 17, 19 and 21 layers using the tight-binding (TB) model without (with) additional bandgap-dependent dielectric screening effect. Dark cyan dots: the measured bandgap tuning for a $10 \mathrm{~nm}$-thick BP film ( 19 layers). The error bars show the s.d., which accounts for the uncertainty in determination of displacement field due to the minor shift of $V_{T M}$ at different temperatures.

because DFT is known for its deficiency to capture screening effects between electrons and subsequent dielectric function. Unlike the case of $4 \mathrm{~nm}$ samples, in which the bandgap variation $(\sim 75 \mathrm{meV})$ is smaller compared with the bandgap itself (the measured bandgap of $4 \mathrm{~nm}$-thick $\mathrm{BP}$ is in fact around $470 \mathrm{meV}$ as shown in Supplementary Fig. 6), the widely changed bandgap in the $10 \mathrm{~nm} \mathrm{BP}$ makes it necessary to include the variation of dielectric screening beyond DFT. Using the randomphase approximation ${ }^{20}$, in which the dominant contribution to the dielectric constant is from the interband transition around the bandgap, we introduced a gap-dependent dielectric constant $\varepsilon\left(E_{\mathrm{g}}\right)=\varepsilon_{0} \frac{E_{\mathrm{g} 0}}{E_{\mathrm{g}}}$ in our tight-binding model, where $\varepsilon_{0}$ is the dielectric constant of pristine $\mathrm{BP}, E_{\mathrm{g} 0}$ is the bandgap of pristine BP ( $~ 300 \mathrm{meV}$ in $10 \mathrm{~nm}$-thick film) and $E_{\mathrm{g}}$ is the bandgap under bias, which can be obtained self-consistently. This model reflects the enhanced dielectric screening when the bandgap is reduced. We have self-consistently calculated bandgaps for BP with thickness from 17 to 21 layers and the results are also plotted in Fig. 4c. The detailed calculation procedures are also presented in Supplementary Note 2. The measured bandgap of $10 \mathrm{~nm}$-thick BP under bias in general agrees well with the self-consistent tight-binding model taking into account the additional dielectric screening. At vertical displacement field above $0.8 \mathrm{~V} \mathrm{~nm}^{-1}$, it seems that the theoretical results underestimate the bandgap reduction, probably due to the oversimplified model for the dielectric constant. Moreover, the measured bandgap tuning results show slight asymmetry as the displacement field switches direction. This is because there are minor uncertainties in the determination of both the bandgap itself and the displacement field. The curve fitting process leads to minor errors in bandgap determination. Moreover, due to the minor device hysteresis in gate bias scans, there is some uncertainty in the determination of displacement field, as indicated by the error bars in Fig. 4c.

\section{Discussion}

In principle, the bandgap tuning effect can be even larger in thicker BP thin films. However, the physical picture above is only valid when the free carrier screening length is longer or comparable to the thickness of BP thin film. In this case, the top and back biases at opposite polarities can effectively compensate the free carriers introduced by each other, leading to an insulating state. In the Supplementary Note 4 and Supplementary Fig. 7, we plot the measured conductance of a $23 \mathrm{~nm}$-thick BP film using the four-probe scheme. It is clear that the doping compensation picture described above does not apply to such thick BP films. As shown in the Supplementary Note 4 and Supplementary Fig. 7, there the top and back gates largely modify the conductance of the top and bottom BP channels, respectively. Therefore, the internal electric field is no longer uniform; the field-induced electrons and holes near the surfaces $^{21}$ will screen the applied electric field. The linear relationship between $V_{\mathrm{TM}}$ and $V_{\mathrm{BG}}$ no longer exists and the thick BP film can no longer be regarded as a material system with a universal bandgap. For BP thinner than $4 \mathrm{~nm}$, the bandgap tuning effect is in fact very weak, as shown in Supplementary Note 5 and Supplementary Fig. 8 in which the results on a $\sim 2.5 \mathrm{~nm}$-thick BP under bias are presented. As a result, a film thickness of $10-15 \mathrm{~nm}$ may be optimal for optoelectronic applications. The required field to tune the bandgap of thinner samples can be considerably larger and thinner samples interact with light less strongly. On the contrary, in thicker samples free carrier screening can render the electric field within BP non-uniform, leading to reduced tuning efficiency.

We want to emphasize that this work represents the demonstration of bandgap tuning in thin-film layered materials whose untuned properties are close to the bulk limit. This is particularly important for future device applications, as thin-film materials interact with light more strongly compared with mono- 
or few-layer materials and the carrier transport in thin film is much less susceptible to environment. Moreover, tuning of bandgap in thin-film BP exhibits distinctively different properties compared with widely explored TMDC bilayers ${ }^{22-24}$. In bilayer TMDCs such as $\mathrm{MoS}_{2}$, due to the weak interlayer electronic state coupling, the bandgap can be tuned effectively and linearly with biasing field, as the interlayer potential difference induced by the external field dominates even in bilayer TMDCs ${ }^{24}$. Although vertical electric field changes the bandgap, the optical transitions are hardly affected by the tuning 24 . That is because the wave functions of the conduction band minimum and the valence band maximum are largely separated by the vertical electric field due the weak interlayer coupling in bilayer $\mathrm{MoS}_{2}$, leading to the much reduced optical transition matrix elements. The optical measurement primarily picks up the transition between the conduction and valence states localized in the same layer, and this energy is nearly fieldindependent. On the contrary, BP is a direct bandgap material regardless of its layer number or applied field. Moreover, the wave functions of the conduction band minimum and valence band maximum of thin-film BP are expected to exhibit substantial overlap even under bias due to the strong interlayer electronic state coupling. This character is of particular importance for optical properties, because the overlap gives rise to significant dipole oscillator strength, implying that the optical emission/ absorption at the direct bandgap edge can be tuned by the gatingfield approach.

In fact, even before the exploration of bilayer TMDs, bandgap opening in bilayer graphene using a vertical electric field has been extensively studied ${ }^{25-33}$. Here the tuning of the bandgap of BP also has distinctively different properties if compared with bilayer graphene. First, it is not feasible to realize bandgap tuning in thinfilm graphite due to strong free carrier screening effect, arising from its metallic nature. Moreover, in general experimentally measured transport bandgaps in biased bilayer graphene are much less than the theoretical predictions and the values determined by optical experiments ${ }^{29-33}$. This is mainly because the spatial fluctuations of the gating field due to the interfacial charges and charges trapped in gate dielectrics lead to some ungated or weakly gated areas ${ }^{33}$. Although they probably represent only a small fraction of the bilayer sample area, these ungated or weakly gated areas are close to metallic and have large conductance. As a result, carrier transport is significantly affected by these highly conductive 'leakage' paths ${ }^{33}$, leading to discrepancy in theory and experiments. In BP thin film, although it is highly likely that there are also such ungated or weakly gated areas, those areas are semiconducting and the transport properties under the bias are dominated by the efficiently gated areas in which the bandgap is significantly reduced. As a result, our transport measurement results agree with theoretical values well.

Finally, we want to comment on the possible role of interfacial state density $\left(D_{\mathrm{it}}\right)$ on our bandgap measurement results. As discussed in Supplementary Note 6 and Supplementary Fig. 9, the interface states can affect the subthreshold slope significantly. However, as long as those states do not participate in current transport themselves, they do not affect the minimum conductivity. As a result, the $D_{\text {it }}$ has negligible effect on the bandgap determination in this work.

In summary, we demonstrate the unique bandgap tuning properties of thin-film BP and further show that the bandgap of a $10 \mathrm{~nm}$-thick BP can be continuously tuned from $\sim 300 \mathrm{meV}$ to below $50 \mathrm{meV}$, with a moderate field readily achieved using regular dielectrics. This demonstration may significantly extend the operational wavelength range of $\mathrm{BP}$ optoelectronic devices, making it a technologically relevant material for tunable infrared applications beyond the cutoff wavelength of pristine BP $(\sim 3.8 \mu \mathrm{m})$. The concept of bandgap tuning may also be leveraged to construct novel electronic devices. Interestingly, this demonstration may further enable the exploration of electrically tunable, BP-based topological insulators and topological nodal semimetals ${ }^{17,18,34}$.

\section{Methods}

Fabrication of dual-gate BP transistors. The fabrication of the devices started with the exfoliation of BP thin films from bulk crystals onto $90 \mathrm{~nm} \mathrm{SiO}_{2}$ on a silicon substrate in an argon-filled glovebox with both oxygen and water concentrations well below one part per million (1 p.p.m.). The thickness of BP flake was determined by AFM and high-resolution transmission electron microscopy measurements. The poly (methyl methacrylate) resist layer was spun on wafer and then patterned for metallization using a Vistec $100 \mathrm{kV}$ electron-beam lithography system. Chromium/Gold $(3 / 30 \mathrm{~nm})$ were then evaporated and the following lift-off process in acetone formed the source, drain and voltage probe contacts on BP flakes. The $24 \mathrm{~nm}$-thick $\mathrm{Al}_{2} \mathrm{O}_{3}$ top-gate dielectric was formed by atomic layer deposition at $150^{\circ} \mathrm{C}$. Titanium/Platinum $(1 / 10 \mathrm{~nm})$ top-gate electrodes were fabricated using the same metallization procedure as discussed above. The top-gate electrode was designed to cover the entire transistor to ensure the full control of the channel.

Conductance measurements using four-probe scheme. All the electrical measurements were performed using an Agilent B1500A semiconductor parameter analyser together with a Keithley $2612 \mathrm{~B}$ source meter in a Lakeshore cryogenic probe station with six probe arms. The static back-gate biases were applied using the source meter, whereas the conductance as a function of top gate bias was measured using the Agilent parameter analyser. The drain bias was set to be as low as $100 \mathrm{mV}$, to minimize the drain-induced doping non-uniformity across the channel, thus ensuring the accurate determination of the conductance minima. The conductance of BP between probes 1 and 2 was obtained through dividing the channel current by the voltage drop between them. The impact of the contact resistance is eliminated by this four-probe scheme. These $\mathrm{Al}_{2} \mathrm{O}_{3}$-covered $\mathrm{BP}$ transistors showed excellent stability. In fact, the measurements usually involved hundreds of scans and multiple temperature cycles. Most of the devices remained operational without noticeable performance degradation after the measurements.

Data availability. The data that support the findings of this study are available from the corresponding authors upon request.

\section{References}

1. Morita, A. Semiconducting black phosphorus. Appl. Phys. A 39, 227-242 (1986).

2. Li, L. et al. Black phosphorus field-effect transistors. Nat. Nanotechnol. 9, 372-377 (2014).

3. Xia, F., Wang, H. \& Jia, Y. Rediscovering black phosphorus as an anisotropic layered material for optoelectronics and electronics. Nat. Commun. 5, 4458 (2014).

4. Liu, H. et al. Phosphorene: an unexplored 2D semiconductor with a high hole mobility. ACS Nano 8, 4033-4041 (2014).

5. Koenig, S. P., Doganov, R. A., Schmidt, H., Neto, A. C. \& Oezyilmaz, B. Electric field effect in ultrathin black phosphorus. Appl. Phys. Lett. 104, 103106 (2014).

6. Castellanos-Gomez, A. et al. Isolation and characterization of few-layer black phosphorus. 2D Mater. 1, 025001 (2014).

7. Qiao, J., Kong, X., Hu, Z.-X., Yang, F. \& Ji, W. High-mobility transport anisotropy and linear dichroism in few-layer black phosphorus. Nat. Commun. 5, 4475 (2014)

8. Rodin, A. S., Carvalho, A. \& Castro Neto, A. H. Strain-induced gap modification in black phosphorus. Phys. Rev. Lett. 112, 176801 (2014).

9. Tran, V., Soklaski, R., Liang, Y. \& Yang, L. Layer-controlled band gap and anisotropic excitons in few-layer black phosphorus. Phys. Rev. B 89, 235319 (2014).

10. Ling, X., Wang, H., Huang, S., Xia, F. \& Dresselhaus, M. S. The renaissance of black phosphorus. Proc. Natl Acad. Sci. USA 112, 4523-4530 (2015).

11. Novoselov, K. S. et al. Two-dimensional gas of massless Dirac fermions in graphene. Nature 438, 197-200 (2005).

12. Zhang, Y., Tan, Y. W., Stormer, H. L. \& Kim, P. Experimental observation of the quantum Hall effect and Berry's phase in graphene. Nature 438, 201-204 (2005).

13. Wang, Q. H., Kalantar-Zadeh, K., Kis, A., Coleman, J. N. \& Strano, M. S. Electronics and optoelectronics of two-dimensional transition metal dichalcogenides. Nat. Nanotechnol. 7, 699-712 (2012).

14. Guo, H., Lu, N., Dai, J., Wu, X. \& Zeng, X. C. Phosphorene nanoribbons, phosphorus nanotubes, and van der Waals multilayers. J. Phys. Chem. C 118, 14051-14059 (2014). 
15. Li, Y., Yang, S. \& Li, J. Modulation of the electronic properties of ultrathin black phosphorus by strain and electrical field. J. Phys. Chem. C 118, 23970-23976 (2014).

16. Dai, J. \& Zeng, X. C. Bilayer phosphorene: effect of stacking order on bandgap and its potential applications in thin-film solar cells. J. Phys. Chem. Lett. 5, 1289-1293 (2014).

17. Liu, Q., Zhang, X., Abdalla, L. B., Fazzio, A. \& Zunger, A. Switching a normal insulator into a topological insulator via electric field with application to phosphorene. Nano Lett. 15, 1222-1228 (2015).

18. Kim, J. et al. Observation of tunable band gap and anisotropic Dirac semimetal state in black phosphorus. Science 349, 723-726 (2015).

19. Sze, S. M. \& Ng, K. K. Physics of Semiconductor Devices. Chapter 1 (John Wiley \& Sons, 2007).

20. Mahan, G. D. Many-Particle Physics 3rd edn Chapter 5.7 (Kluwer Academic/ Plenum Publishers.

21. Low, T. et al. Plasmons and screening in monolayer and multilayer black phosphorus. Phys. Rev. Lett. 113, 106802 (2014)

22. Ramasubramaniam, A., Naveh, D. \& Towe, E. Tunable band gaps in bilayer transition-metal dichalcogenides. Phys. Rev. B 84, 205325 (2011).

23. Koskinen, P., Fampiou, I. \& Ramasubramaniam, A. Density-functional tightbinding simulations of curvature-controlled layer decoupling and band-gap tuning in bilayer $\mathrm{MoS}_{2}$. Phys. Rev. Lett. 112, 186802 (2014).

24. Chu, T., Ilatikhameneh, H., Klimeck, G., Rahman, R. \& Chen, Z. Electrically tunable bandgaps in bilayer $\mathrm{MoS}_{2}$. Nano Lett. 15, 8000-8007 (2015).

25. McCann, E. Asymmetry gap in the electronic band structure of bilayer graphene. Phys. Rev. B 74, 161403 (R) (2006).

26. Castro, E. V. et al. Biased bilayer graphene: semiconductor with a gap tunable by the electric field effect. Phys. Rev. Lett. 99, 216802 (2007).

27. Min, H., Sahu, B., Banerjee, S. K. \& MacDonald, A. H. Ab initio theory of gate induced gaps in graphene bilayers. Phys. Rev. B 75, 155115 (2007).

28. Ohta, T., Bostwick, A., Seyller, T., Horn, K. \& Rotenberg, E. Controlling the electronic structure of bilayer graphene. Science 313, 951-954 (2006).

29. Oostinga, J. B., Heersche, H. B., Liu, X., Morpurgo, A. F. \& Vandersypen, L. M. K. Gate-induced insulating state in bilayer graphene devices. Nat. Mater. 7, 151-157 (2008).

30. Zhang, Y. et al. Direct observation of a widely tunable bandgap in bilayer graphene. Nature 459, 820-823 (2009).

31. Mak, K. F., Lui, C. H., Shan, J. \& Heinz, T. F. Observation of an electric-fieldinduced band gap in bilayer graphene by infrared spectroscopy. Phys. Rev. Lett. 102, 256405 (2009).

32. Xia, F., Farmer, D. B., Lin, Y. -M. \& Avouris, P. Graphene field-effect transistors with high on/off current ratio and large transport band gap at room temperature. Nano Lett. 10, 715-718 (2010).

33. Chu, T. \& Chen, Z. Achieving large transport bandgaps in bilayer graphene. Nano Res. 8, 3228-3236 (2015).

34. Yang, B.-J. \& Nagaosa, N. Classification of stable three-dimensional Dirac semimetals with nontrivial topology. Nat. Commun. 5, 4898 (2014).

\section{Acknowledgements}

F.X. acknowledges the financial support through a Young Investigator Program (YIP) from the Office of Naval Research (N00014-15-1-2733). L.Y., H.W. and J.C. thank the support from the National Science Foundation EFRI-2DARE programme (EFMA-1542815). Facilities use in Yale was partially supported by Yale Institute for Nanoscience and Quantum Engineering (YINQE) and NSF MRSEC DMR 1119826. S.J.K. was supported in part by the National Science Foundation through the University of Minnesota MRSEC under Award Number DMR1420013. We acknowledge the Imaging Facility of CUNY Advanced Science Research Center for instrument use, scientific and technical assistance. We thank Professor Wang Yao at Hong Kong University and Dr Xiaolong Chen at Yale University for insightful comments.

\section{Author contributions}

L.Y. and F.X. conceived and supervised the project. B.D., H.J., C.L., H.T., Q.X. and H.W. participated in device fabrication. B.D., Q.G. and X.W. performed device characterizations. Y.X. and J.C. performed the device cross-section characterizations. V.T. and L.Y. did the modelling. S.J.K. performed the analysis of the impact of the interfacial states on the minimum conductivity. F.X., L.Y., B.D. and V.T. drafted the manuscript. All the authors discussed the results and commented on the manuscript.

\section{Additional information}

Supplementary Information accompanies this paper at http://www.nature.com/ naturecommunications

Competing interests: The authors declare no competing financial interests.

Reprints and permission information is available online at http://npg.nature.com/ reprintsandpermissions/

How to cite this article: Deng, B. et al. Efficient electrical control of thin-film black phosphorus bandgap. Nat. Commun. 8, 14474 doi: 10.1038/ncomms14474 (2017).

Publisher's note: Springer Nature remains neutral with regard to jurisdictional claims in published maps and institutional affiliations.

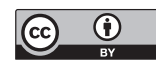

This work is licensed under a Creative Commons Attribution 4.0 International License. The images or other third party material in this article are included in the article's Creative Commons license, unless indicated otherwise in the credit line; if the material is not included under the Creative Commons license, users will need to obtain permission from the license holder to reproduce the material. To view a copy of this license, visit http://creativecommons.org/licenses/by/4.0/

(C) The Author(s) 2017 